\title{
25595
}

\section{Challenges and Pitfalls in Multi-Physics Integration for Reservoir Characterisation}

L. MacGregor* (RSI), J. Tomlinson (RSI) \& R. Cooper (RSI)

\section{SUMMARY}

Combining multiple geophysical data types using integrated interpretation or joint inversion approaches can provide information on earth properties that is either unreliable or simply unavailable when only a single data type is considered. In particular the combination of seismic, CSEM and well log data has the potential to improve the certainty with which reservoir lithology and fluid properties are constrained. 


\section{Introduction}

Combining multiple geophysical data types using integrated interpretation or joint inversion approaches can provide information on earth properties that is either unreliable or simply unavailable when only a single data type is considered. In particular the combination of seismic, CSEM and well $\log$ data has the potential to improve the certainty with which reservoir lithology and fluid properties are constrained.

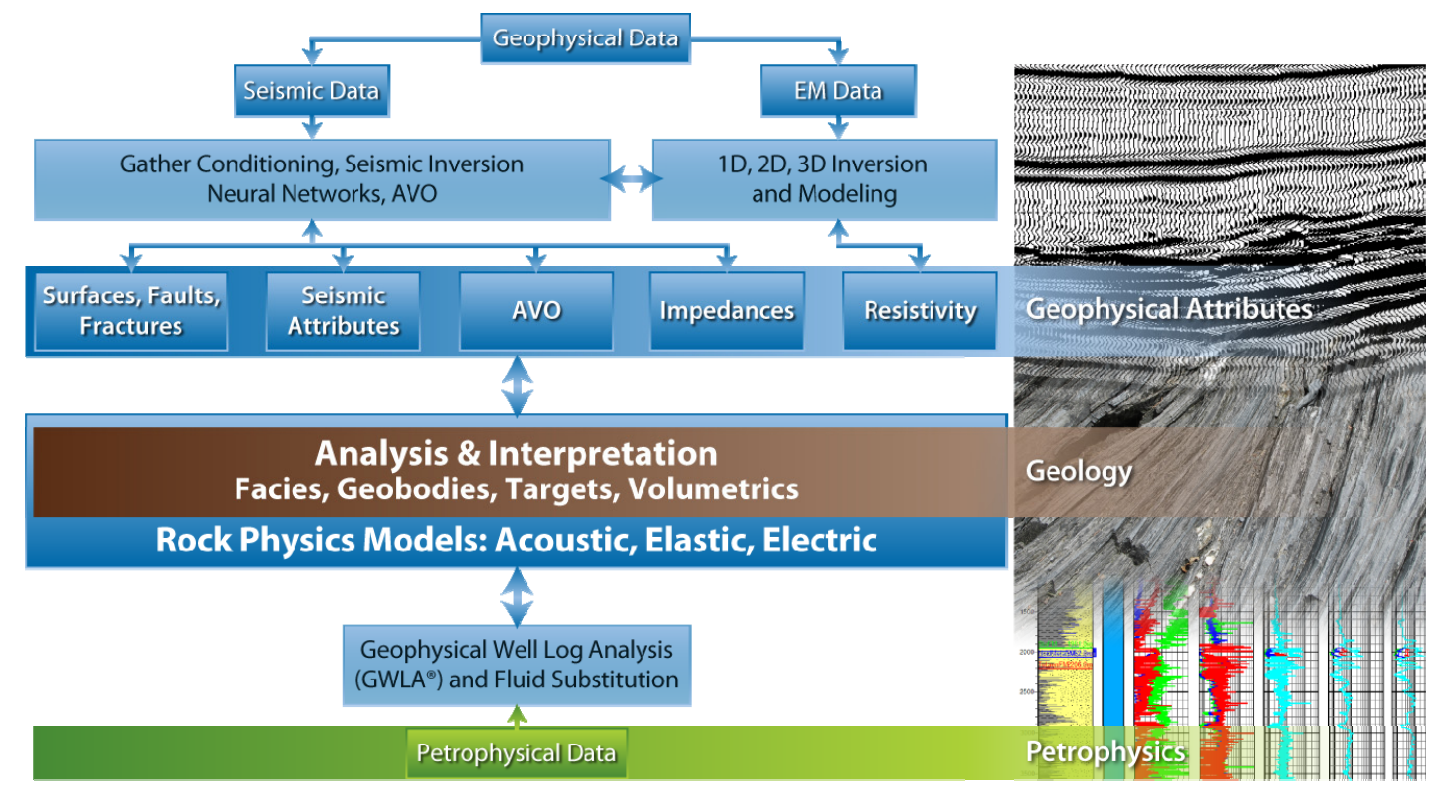

Figure 1 An overview of an integrated interpretation workflow applied to seismic, well log and CSEM data. The goal of this process is to develop a geologically sound earth model consistent with all of the data types used in the analysis

\section{Integration Considerations}

Although integrated interpretation brings many benefits, there are a number of challenges to be overcome before such approaches can be robustly applied. Firstly measurements made using very different physical processes (electric and elastic in the case of CSEM and seismic) must be combined and linked to the underlying rock and fluid properties in a consistent fashion. This requires a rock physics framework to be either numerically derived or empirically calibrated at well locations. In both cases such models are subject to uncertainty, which in turn leads to uncertainty in the resulting interpretation.

Secondly seismic, CSEM and well log techniques sample the earth at very different scales, varying from a few $\mathrm{cm}$ in the case of well logs, to hundreds of metres for CSEM. These different scales must be reconciled in an integrated interpretation or joint inversion approach.

Finally in order for an integrated interpretation approach to be successful, both seismic and CSEM methods must be sensitive to the interval of interest and changes in properties within it. Although this is perhaps an obvious statement, it is however a key consideration in determining where such approaches can be applied. 


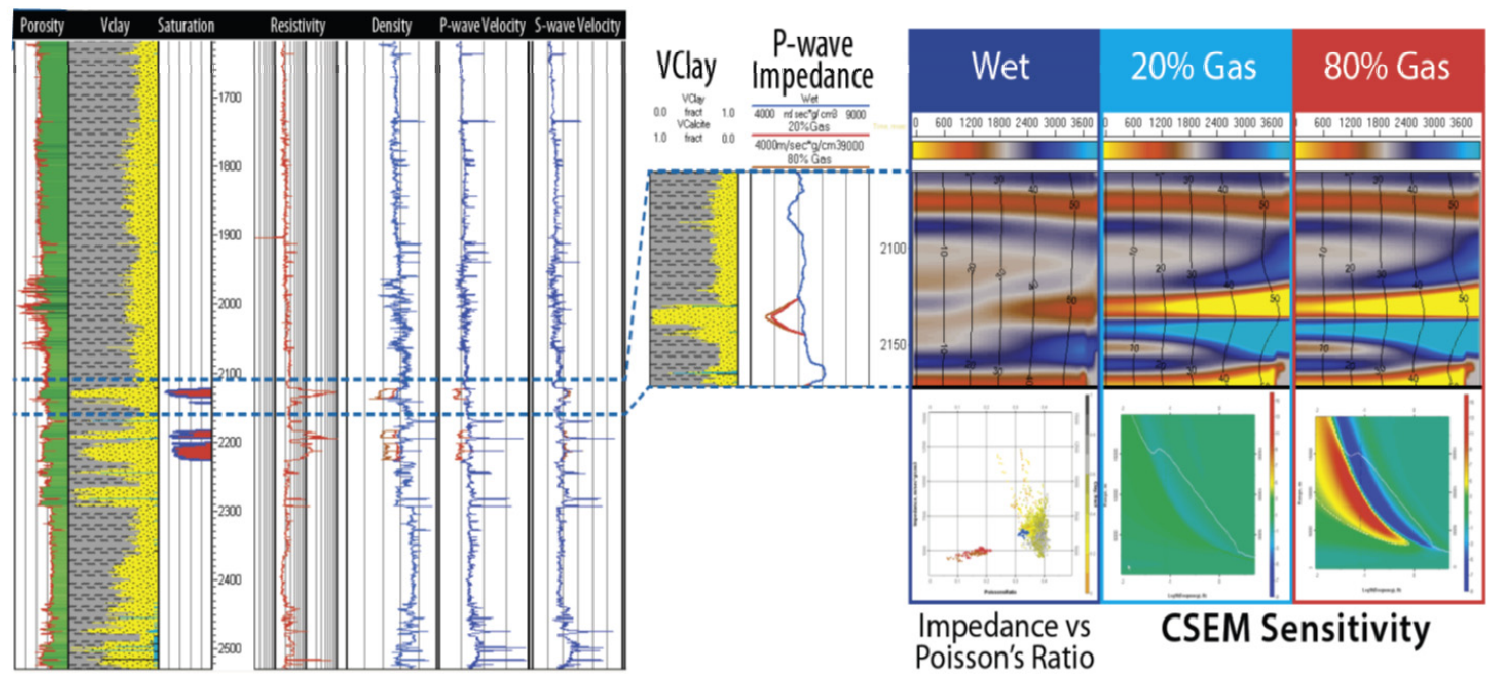

Figure 2 An example of rock physics modelling to understand the effect of varying fluid type and saturation on the elastic and electric responses. On the left a consistent petrophysical interpretation is computed for each well, including mineral and fluid volumetrics.

\section{Conclusions}

The solutions to these challenges are case dependent and must be considered with care. For any given geophysical question, the most robust answer will be obtained by using the tool, or combination of tools best suited to the task, and determining this combination is the first step in any analysis. The resulting choice of data must then be integrated within a rock physics framework, to provide a model that is geologically reasonable, and consistent with each of the geophysical data types available.

\section{Acknowledgements}

The authors would like to thank the many staff from RSI who have over a number of years developed, tested and modified the workflow that will be discussed in this paper. 